La evolución de la crisis del COVID en las portadas de la prensa española de referencia

Francisco Leslie López del Castillo Wilderbee

Question/Cuestión, Nro.69, Vol.3, agosto 2021

ISSN: 1669-6581

URL de la Revista: https://perio.unlp.edu.ar/ojs/index.php/question/

IICom -FPyCS -UNLP

DOI: https//doi.org/10.24215/16696581e571

\title{
La evolución de la crisis del COVID en las portadas de la prensa española de referencia
}

The evolution of the COVID crises on the front pages of the leading Spanish press

\section{Francisco Leslie López del Castillo Wilderbeek}

Licenciado en Comunicación Audiovisual por la Universtitat Oberta de Catalunya.

Doctor en comunicación por la Universitat Pompeu Fabra y miembro del equipo de

investigación Medium

Universitat Oberta de Catalunya

España

franciscoleslie@alumni.upf.edu

http://orcid.org/0000-0002-6664-7849

\section{Resumen}

Esta investigación ha analizado el tratamiento de la crisis del Covid en los titulares de los temas principales de portada de los tres diarios con mayor público en España. Mediante análisis de 
contenido y visualización de datos se ha buscado la existencia de correlaciones entre cobertura informativa y número de contagios, y si se producen cambios de tendencias informativas entre la primera y la segunda ola de la pandemia. Las conclusiones finales señalan que los diarios analizados muestran un desgaste potencial en el tratamiento informativo de la pandemia además de poner el foco en las normas que condicionan el estilo de vida.

Palabras clave: covid, pandemia, noticias, prensa, portadas

\section{Abstract}

This research has analyzed the coverage of the Covid crisis in the main front page headlines of the three newspapers with the largest audience in Spain. Applying content analysis and data visualization, we have searched for correlations between news coverage and number of contagions, and whether there are changes in news trends between the first and second wave of the pandemic. The final conclusions point out that the analyzed newspapers show a relaxation in the informative treatment of the pandemic in addition to focusing on the rules that determine the lifestyle.

Keywords: covid, pandemic, news, press, front page, front pages

\section{INTRODUCCIÓN}

La pandemia por el COVID ha producido un impacto determinante en la vida diaria de los ciudadanos de todo el planeta, esta situación ha afectado con similar intensidad a la actividad de los medios de comunicación. Como señalan Lázaro-Rodríguez y Herrera-Viedma (2020: 2) además de la información de tipo científico durante el azote de la pandemia: "cabe destacar el papel de la información difundida por medios de difusión impresos y digitales". Prueba de ello es que además de la producción de información (Aleixandre-Benavent, Castelló-Cogollos y Valderrama-Zurián, 2020) durante la primera fase de la pandemia en España el consumo de noticias aumentó en un $62 \%$ entre la audiencia menos habituada a los productos informativos (Casero-Ripollés, 2020).

Este mismo fenómeno impelió al Gobierno español como fuente principal de información con el propósito de minimizar la infodemia' (Castillo-Esparcia, Fernández-Souto y Puentes-Rivera, 
2020). Ante esta situación la credibilidad de los medios de comunicación también ha sido una columna fundamental para ofrecer confianza a los públicos. Tal como acertadamente señala Xifra (2020) ante la pandemia los medios de comunicación tradicionales han ejercido de medios refugio para la ciudadanía según los datos de percepción entre esta (Edelman, 2020)ii. Dentro de este concepto de medios de refugio se encuentra un nicho más específico compuesto por los diarios de referencia conocidos como quality papers (Alvarado, 2008). Las portadas de estos medios se encuentran dotadas de una importancia capital para ofrecer información contrastada a sus públicos, expresando el enfoque informativo que es trasladado a la audiencia y son un indicador a largo plazo sobre el desarrollo de las temáticas que tienen cabida (Danielson y Lasorsa, 2020), como podría ser la crisis por el COVID.

Este trabajo ha tenido el objetivo de analizar los titulares de los temas principales de las portadas de los tres diarios españoles con mayor público: El paísiii, El mundo ${ }^{\mathrm{iv}}$ y La Vanguardia ${ }^{v}$ entendidos como medios refugio (Xifra, 2020) y más concretamente quality papers (Alvarado, 2008) durante la primera y segunda ola de contagios de la pandemia.

\section{METODOLOGÍA}

El marco temporal de este análisis ha comprendido desde que la pandemia por el COVID empezó a afectar a la sociedad de este país (11 de febrero de 2020) ${ }^{\mathrm{vi}}$ hasta el punto álgido de la llamada segunda ola de la pandemia (7 de noviembre de 2020) ${ }^{\text {vii }}$. El acceso a estos contenidos ha sido posible gracias a la consulta a la base de datos de la empresa REBOLD, líder en el análisis de la comunicación.

Conviene reseñar que esta investigación se encuentra firmemente relacionada con los relevantes trabajos de Gómez et al. (2020) $)^{\text {viii }}$ y de Sánchez et al. (2020) . No obstante, aunque presente estudio comparte su mismo punto de partida (análisis de las portadas de diarios de referencia sobre el COVID) existen cambios sustanciales en la metodología y por ende en los resultados como pudiera ser la comparación con los datos médicos de afectación de la pandemia, el uso de la visualización de datos y especialmente la extensión del marco temporal hasta la segunda ola. 
Para el análisis de las portadas se ha aplicado análisis de contenido. El análisis de contenido es una técnica que permite la codificación de las características de los productos difundidos por los medios de comunicación (Macnamara, 2018) de forma objetiva, sistemática (Krippendorff, 2018) y cuantificable (Neuendorf, 2017)

Para la codificación del titular principal en las portadas de los diarios citados se ha aplicado un criterio comparativo basado en el tamaño de la tipografía. Los temas tratados en portada con la tipografía más grande se han considerado temas de portada, el resto con tipografía más pequeña no han sido considerados como el principal de portada y no se han incluido.

Aquellos temas incluidos como principales de portada se han codificado como temática COVID si el titular hacía mención explícita o implícita a la pandemia. En aquellos titulares aceptados en la investigación como categoría COVID se ha aplicado una subcategorización en cuatro temáticas no excluyentes: Sanidad, Política, Economía y Social en sintonía con el trabajo realizado por Abuín, et al. (2020) en medios digitales. Los criterios han sido los siguientes:

Sanidad: El titular presenta datos sobre el alcance de la pandemia y las medidas estrictamente sanitarias (por ejemplo número de muertos o de contagiados, compra de material sanitario, estado de saturación en los hospitales).

Política: El titular hace mención explícita o implícita a acciones o reacciones políticas por la pandemia (por ejemplo anuncios de los diferentes gobiernos o declaraciones de la oposición).

Economía: El titular hace mención explícita o implícita a repercusiones o medidas económicas por la pandemia (por ejemplo fondos públicos de reconstrucción, ERTES o movimientos de la bolsa).

Social: El titular hace mención explícita o implícita a medidas que afectan a la convivencia y el estilo de vida en sociedad (por ejemplo confinamiento, desescalada, estado de alarma, restricciones a la movilidad o disturbios contra la acción del gobierno).

Los datos obtenidos mediante esta categorización se han expresado en formato gráfico para realizar visualización de datos. Esta técnica supera la mera observación (Godino, Gonzato, Cajaraville y Fernández, 2012) y tiene el propósito de extraer conocimiento nuevo (Arcavi, 
2003) mostrando lo que hay más allá de las características generales de los datos (Anscombe, 1973). A su vez la representación gráfica de los resultados obtenidos mediante análisis de contenido se han confrontado con los datos de contagios en España obtenidos gracias al portal de Eurostat. De esta forma se ha podido verificar si puede extraerse algún tipo de correlación entre la cobertura mediática en los medios de referencia citados y la verdadera afectación de la pandemia.

\section{RESULTADOS}

\subsection{Resultados globales}

Aplicando los criterios mencionados anteriormente se obtuvo un total de 810 portadas durante 270 días $^{\mathrm{xi}}$. De esas 810 portadas 529 fueron categorizadas como referidas al COVID lo que conduce a un $65,3 \%$ respecto el total. Tal como se ha comentado dentro de la categorización COVID se generaron subgrupos no excluyentes etiquetados como Política: 371 (70\%), Economía: 167 (31,5\%), Social: 187 (35\%) y Sanidad: 105 (20\%).

Además de esta categorización se analizó la presencia de entidades (términos referentes a personas, organizaciones o lugares) dentro los titulares clasificados como temática COVID. Los términos obtenidos se cuantificaron según la entidad a la que estaban referidos. Por ejemplo los términos Sánchez (en referencia al presidente del Gobierno), Gobierno, Illa, Iglesias y Marlaska (en referencia a los ministros) se codificaron y cuantificaron como referidos a la entidad: Gobierno. El resto de conceptos-entidad detectados fueron: Autonomías (y variantes en genérico), Madrid (Madrid, Ayuso), Catalunya (Catalunya, Govern, Generalitat, Barcelona), Europa (y variantes en genérico) y Oposición (PP, Cs, Vox). 


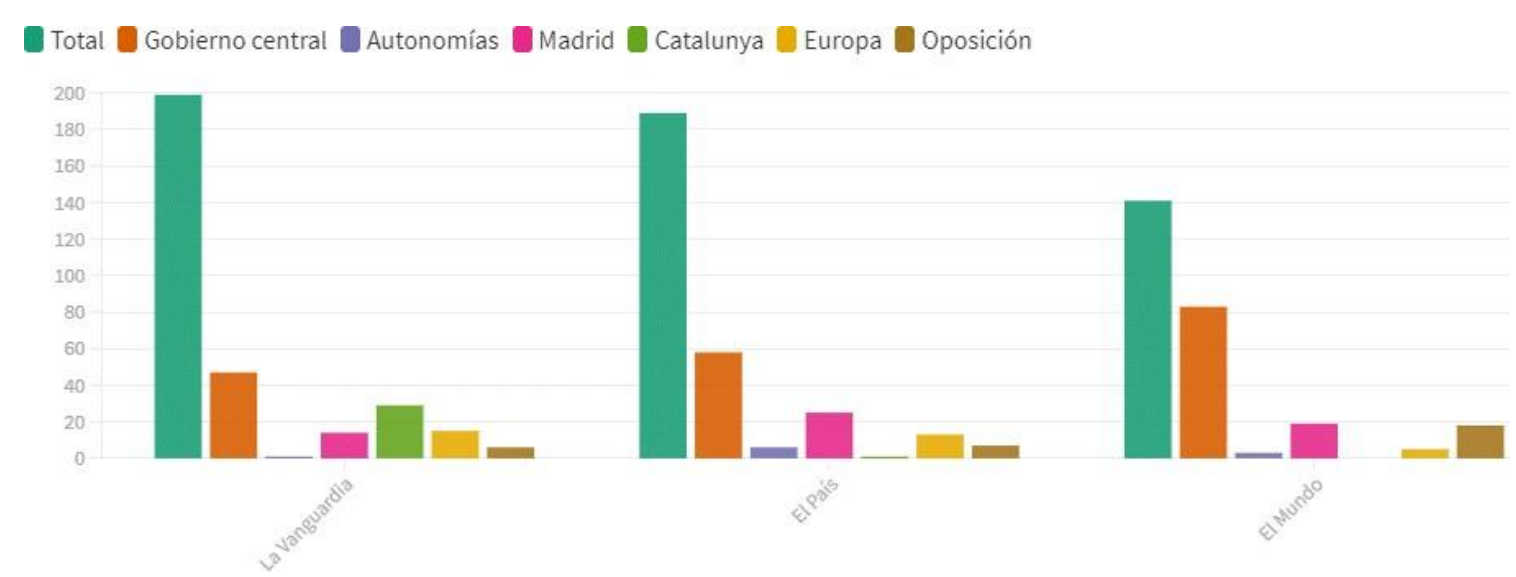

Figura 1. Cuantificación del número de portadas referidas al COVID y de aquellas que mencionan las entidades: Gobierno central, Autonomías, Madrid, Catalunya, Europa y

Oposición. Fuente: propia y Eurostat

Este enfoque descriptivo aporta información que coincide con las propias características de los tres medios incorporados en la investigación. Por ejemplo, La Vanguardia (con sede en Catalunya) realizó una cobertura mayor de la pandemia en Catalunya en comparación con El País y el Mundo cuya sede se encuentra en Madrid ${ }^{\mathrm{xii}}$ tal como puede observarse en la figura 1.

Por otro lado, el Mundo, como medio con menor tasa de portadas incluidas, mostró una elevada proporción de presencia de la entidad Gobierno central y especialmente de la entidad Oposición en comparación con los otros dos medios. A su vez, la presunta perspectiva crítica que puede extraerse de la importante presencia de partidos de la oposición en el tratamiento informativo podría correlacionarse con que fue el único medio que mencionó en titular de portada al Ministro del Interior Fernando Grande-Marlaska. En relación con la presencia de esta entidad el Mundo fue el único medio que colocó de forma negativa xiii como tema de portada la investigación sobre la manifestación del 8-M en la que se cuestionó que el Gobierno promoviera un acto multitudinario cuando ya existían sospechas sobre la potencial propagación del COVID en España ${ }^{\text {xiv }}$. Este segundo indicio sobre el tratamiento negativo junto con el protagonismo de los partidos de oposición podría estar conectado a la situación de pandemia o a la propia línea editorial del medio ${ }^{x v}$. Esta segunda opción confirmaría indirectamente la 
opinión de algunos autores sobre si existe un sesgo político que condiciona la cobertura en algunos medios (Masip et al., 2020; Motta, Stecula y Farhart (2020).

\subsection{Tratamiento informativo y número de contagios}

No obstante, los resultados globales mostrados en el anterior apartado deben considerarse como simples acumulaciones de los datos observados durante la franja analizada y no representan las dinámicas existentes o su potencial correlación con la expansión de la pandemia en territorio español. Para poder desentrañar esta segunda posibilidad se comparó a nivel mensual la producción de portadas cuyo tema principal era la pandemia respecto el total de contagios durante esas mismas fechas.

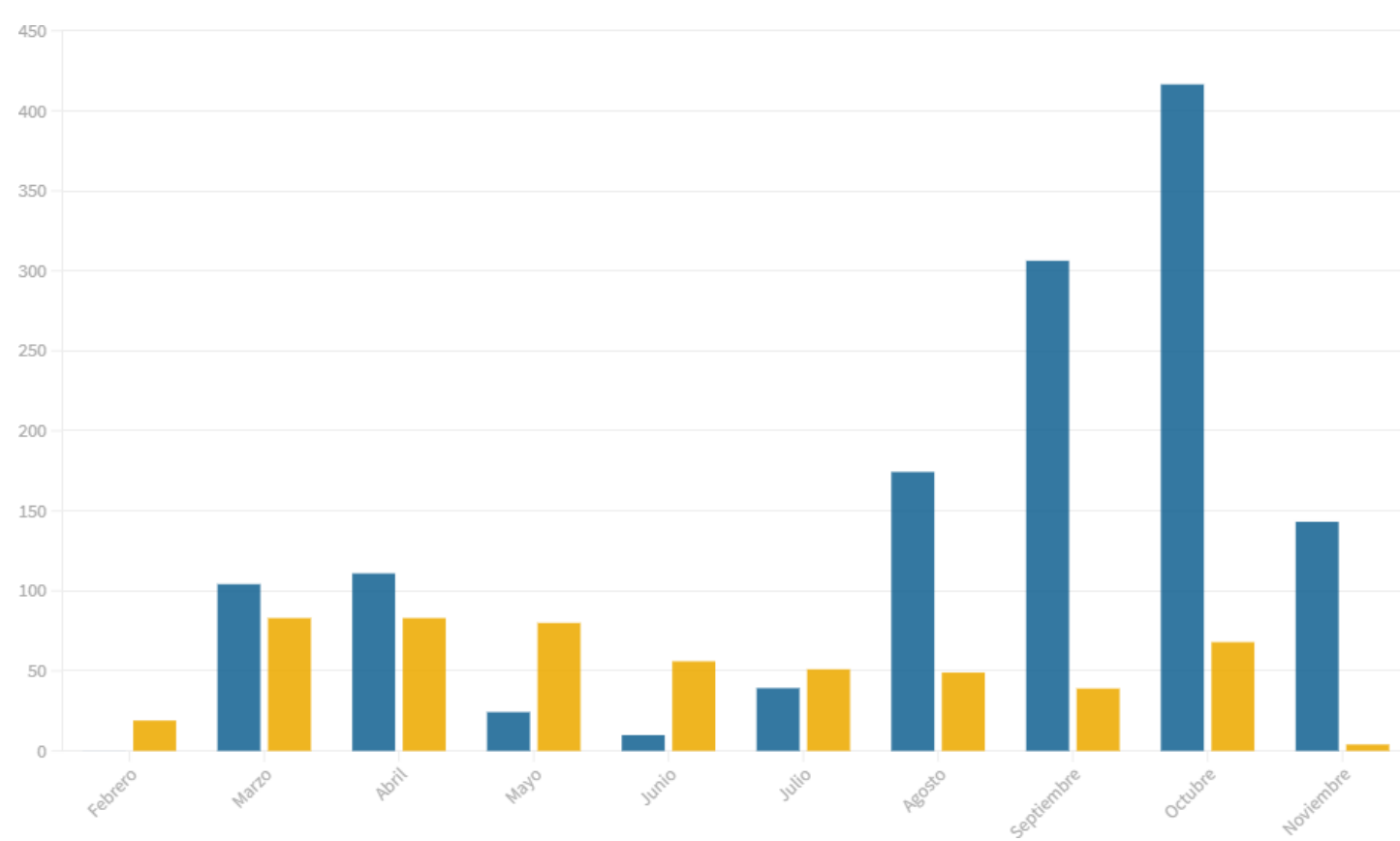

Figura 2. Total de portadas con tema principal dedicado al Covid y total de contagios en España según EU Open Data Portal ${ }^{x v i}$ desde el 11 de febrero hasta el 7 de noviembre de 2020.

La organización se ha realizado por meses y para la comparación se ha aplicado una proporción 1:1.000 (una portada equivale a 1.000 contagios)

Fuente: propia y Eurostat 
Como puede observarse en la Figura 2 en términos proporcionales el número de contagios superó al número de portadas durante los meses con mayor incidencia tanto de la primera como de la segunda ola. Conviene reseñar que aunque el mes de febrero y el de noviembre estén incompletos se han computado en ambos tanto los datos de portadas como de contagios de los días de esos meses que sí han entrado en la investigación.

El primer elemento significativo que puede extraerse de la confrontación entre número de contagios y temas principales de portada es que existe cierta correlación entre aumento de casos y aumento de presencia en portadas. No obstante puede apreciarse que la curva de contagios es mucho más elástica en relación a la curva de portadas sobre el COVID. Esto es debido a que el techo de contenidos categorizados al día es de 3 portadas lo que equivaldría a 3.000 contagios (equivalencia 1:1.000), cifra que desgraciadamente se superó tanto en la primera como especialmente en la segunda ola.

Sin embargo, si tal como puede apreciarse en la gráfica, la prensa se adelantó a la aparición de los primeros contagios en España este fenómeno no se produjo en la segunda ola. Pese a que el número de contagios fue muy superior en la segunda ola no se alcanzaron los niveles de portadas dedicadas al COVID de los meses de marzo y abril (83 portadas de 90 posibles en ambos casos). El nivel máximo de portadas de la segunda ola se produjo en octubre con 68 portadas de 93 posibles cuando los datos de contagios habían superado ostensiblemente a los registrados durante los primeros meses de la pandemia.

Este fenómeno podría explicarse como una potencial normalización de la situación, la visión informativa que se tiene de la pandemia pierde excepcionalidad y deja paso a otros temas principales en las portadas de los diarios más importantes. Pruebas de esto serían la incursión de temas informativos como el llamado "caso Dina"xvii , la monarquía ${ }^{x v i i i}$, el conflicto por la renovación del poder judicial ${ }^{\mathrm{xix}}$, la llamada "trama Gürtel”x $\mathrm{o}$ las elecciones en Estados Unidos $^{x \times i}$. Como puede observarse la situación políticaxxii fue en cierta forma un contrapunto suficiente para desplazar el protagonismo de la pandemia en los citados diarios durante la segunda ola pese a la intensificación del número de contagios.

El segundo elemento que puede extraerse de la confrontación entre temas de portada y número de contagios es que si bien en la primera ola la prensa se adelantó a la aparición de 
los primeros casos en la segunda se observa un comportamiento reactivo. Este indicio se basa en el aumento de portadas computado en octubre, cuando los datos de contagios ya estaban fuera de todo registro conocido.

Finalmente, y vinculado al segundo elemento mencionado, se puede afirmar que pese a que la afectación de la pandemia bajó ostensiblemente durante el final de la primavera y el inicio del verano las portadas incorporadas en esta investigación mantuvieron el interés en ella. Este hallazgo podría definirse como una función de alerta de los medios refugio. Pese a una reducción importante del número de casos los principales diarios siguieron informando y considerándolo una prioridad en sus portadas.

Esta desviación en la correlación puede observarse claramente en la Figura 2. Durante los meses fuera de los picos de la pandemia (mayo, junio, julio) la relación entre volumen de portadas y contagios se subvierte completamente. Conviene reseñar de nuevo que la gráfica está generada en proporción 1:1.000, si bien esta misma diferencia de escala no es obstáculo para que se adviertan diferencias significativas a la inversa en los meses con mayores contagios. Por tanto, puede afirmarse que la prensa de los tres principales diarios españoles mantuvo una función de alerta al conservar la atención en la afectación y peligros de la pandemia pese a los buenos datos de contagios.

\subsection{Temáticas asociadas y alteraciones en las tendencias}

Siguiendo con la metodología explicitada anteriormente se cuantificaron tanto las portadas, como la categorización según temática COVID y dentro de esta las subagrupaciones no excluyentes: Política, Social, Economía y Sanidad. 


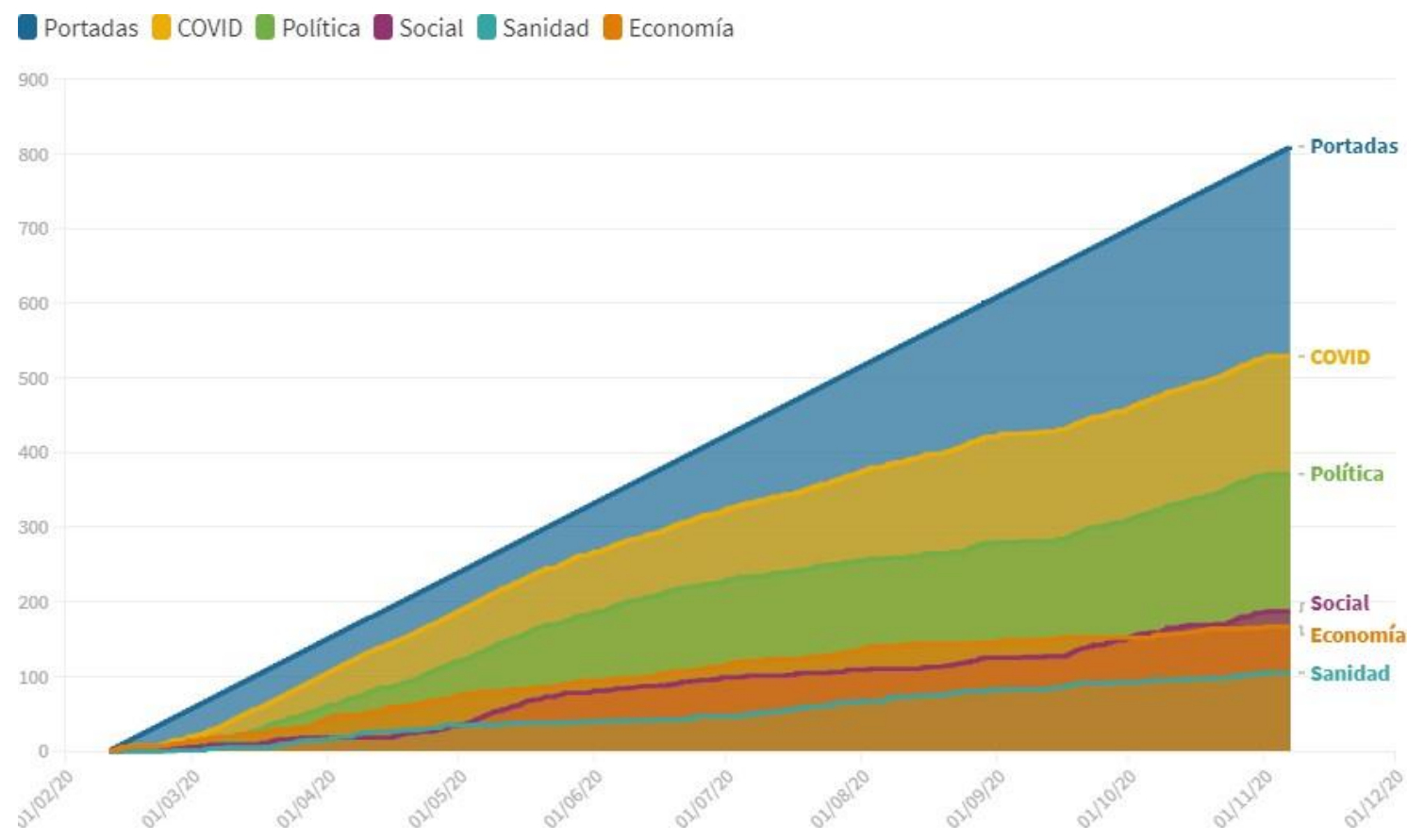

Figura 3. Línea de tiempo con el total de portadas publicadas desde el 11 de febrero hasta el 7 de noviembre y la categorización según tema principal. La agrupación principal fue COVID y dentro de esta: Política, Social, Economía y Sanidad. Fuente: propia

Como puede observarse en la figura 3 durante el inicio de la pandemia la tendencia de generación de portadas con temática COVID se movió en paralelo al total de portadas cuantificadas. Este dato es correlativo con los aumentos de los meses de marzo, abril y mayo presentes en la figura 2. De la misma forma la directriz alcista de la temática COVID empieza a desviarse en el mes de junio respecto todas las portadas posibles. Esta visualización reafirma los datos organizados mensualmente observados en la figura 2 y que señalaban una caída en los temas de portada al finalizar la primera ola.

No obstante la cuestión más relevante que permite observar la organización en línea de tiempo es que esta dinámica en la que el COVID pierde interés como tema principal se mantiene a largo plazo alcanzando la segunda ola y originando una brecha que el repunte de interés informativo de octubre no puede corregir. La visualización de datos permite exponer claramente 
cómo el tratamiento informativo en las portadas, ya no en relación al número de contagios (expresado en la figura 2) sino en el mismo volumen de producción periodística, reduce el interés por el COVID como tema principal. Este resultado reafirmaría la presunción de la normalización de la pandemia en el ámbito informativo en comparación con la hegemonía existente durante los primeros momentos de la crisis. Por otra parte, este cambio en el volumen de cobertura podría correlacionarse indirectamente con los resultados obtenidos por Masip et al. (2020) según los cuales en la primera ola ya se detectaron síntomas de agotamiento entre la audiencia sobre la preeminencia de la temática COVID en los medios.

Por otro lado, el análisis de las temáticas vinculadas a la pandemia muestra también alteraciones respecto el tratamiento en el principio de la pandemia y en las fases posteriores. Estas dos digresiones sobre las tendencias hegemónicas involucran al aumento de la temática Social en comparación con el aumento del resto de temáticas. Conviene recordar que la categorización de un tema de portada como Social se encuentra vinculado a conceptos como el confinamiento, las restricciones a la movilidad o el rechazo de la sociedad a las decisiones del Gobierno.

El aumento de la subagrupación Social es muy relevante sobre todo teniendo en cuenta el estudio de Gómez et al. (2020) pues en ese, y con una metodología similar, se detectó que los tres temas principales durante el inicio de la pandemia fueron Sanidad Política y Economía ${ }^{\text {xiii. }}$ Esta misma estructura, aunque con diferencias de jerarquía, es la presente con la metodología de este trabajo durante las primeras fases de la pandemia, sin embargo, se produce una evolución a lo largo del tiempo hasta introducir a Social como el segundo tema más importante una vez alcanzado el pico de la segunda ola (principios de noviembre).

Los dos cambios de jerarquía pueden observarse en detalle en las figuras 4 y 5 . 


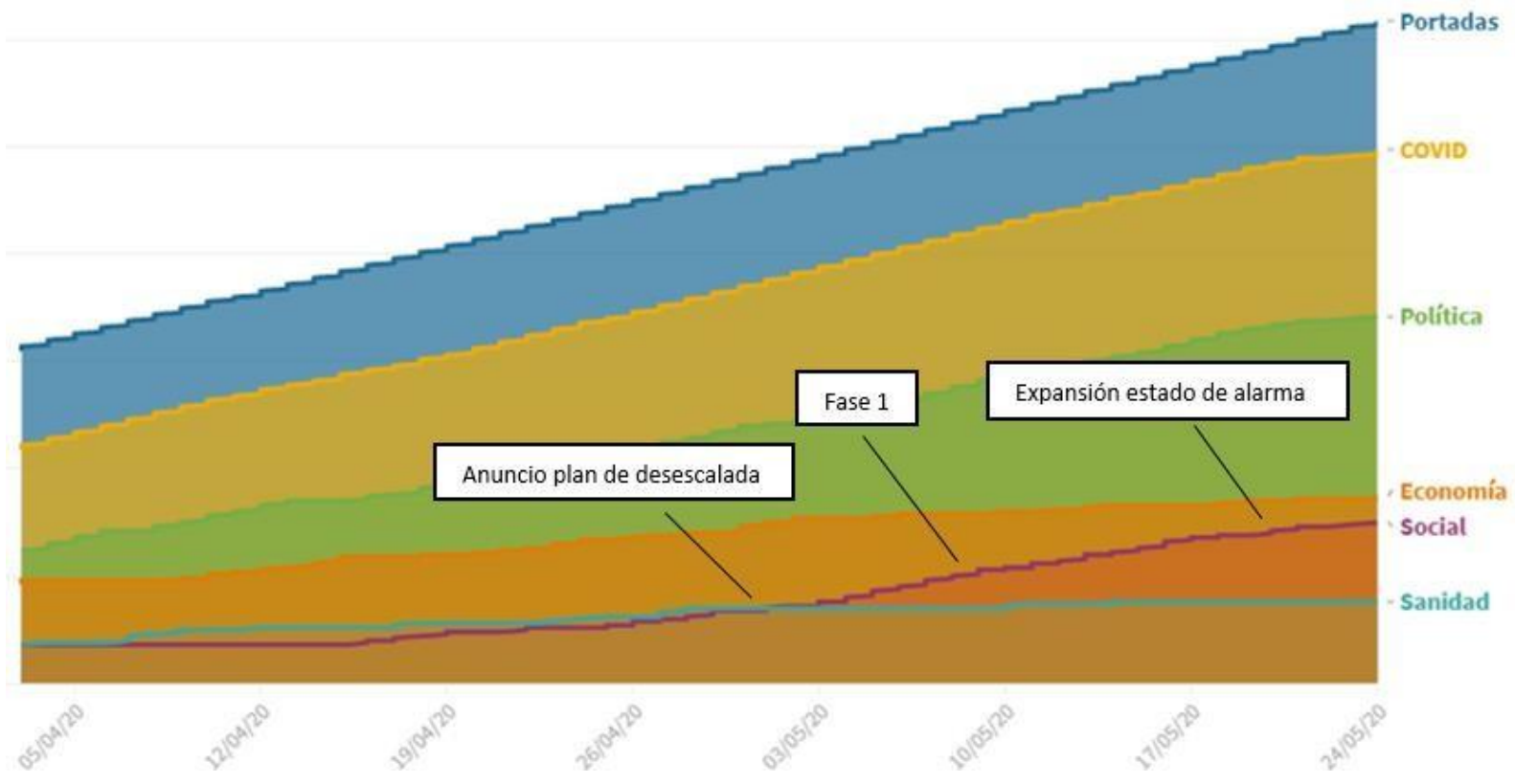

Figura 4. Línea de tiempo con el total de portadas publicadas desde el 1 de abril hasta el 2 de mayo y la categorización según tema principal. La agrupación principal fue COVID y dentro de esta: Política, Social, Economía y Sanidad. Fuente: propia

Como puede observarse en la figura 4 las portadas dedicadas a medidas que afectaban a la vida en sociedad aumentaron de volumen coincidiendo con el anuncio del plan de desescalada llegando a superar a aquellas con información estrictamente sanitaria. Por otro lado, la aplicación de la desescalada por fases aumentó esa brecha que se mantuvo a largo plazo con el anuncio de la ampliación del estado de alarma. Resulta muy significativo que en el momento en que la temática Social superó a la temática Sanidad según los datos de esta investigación se publicara una primera información sobre el presunto desgaste que estaban originando sobre la población la medidas del Gobierno ("La policía se prepara para un aumento de delitos de orden público" El Mundo, 2 de mayo). 


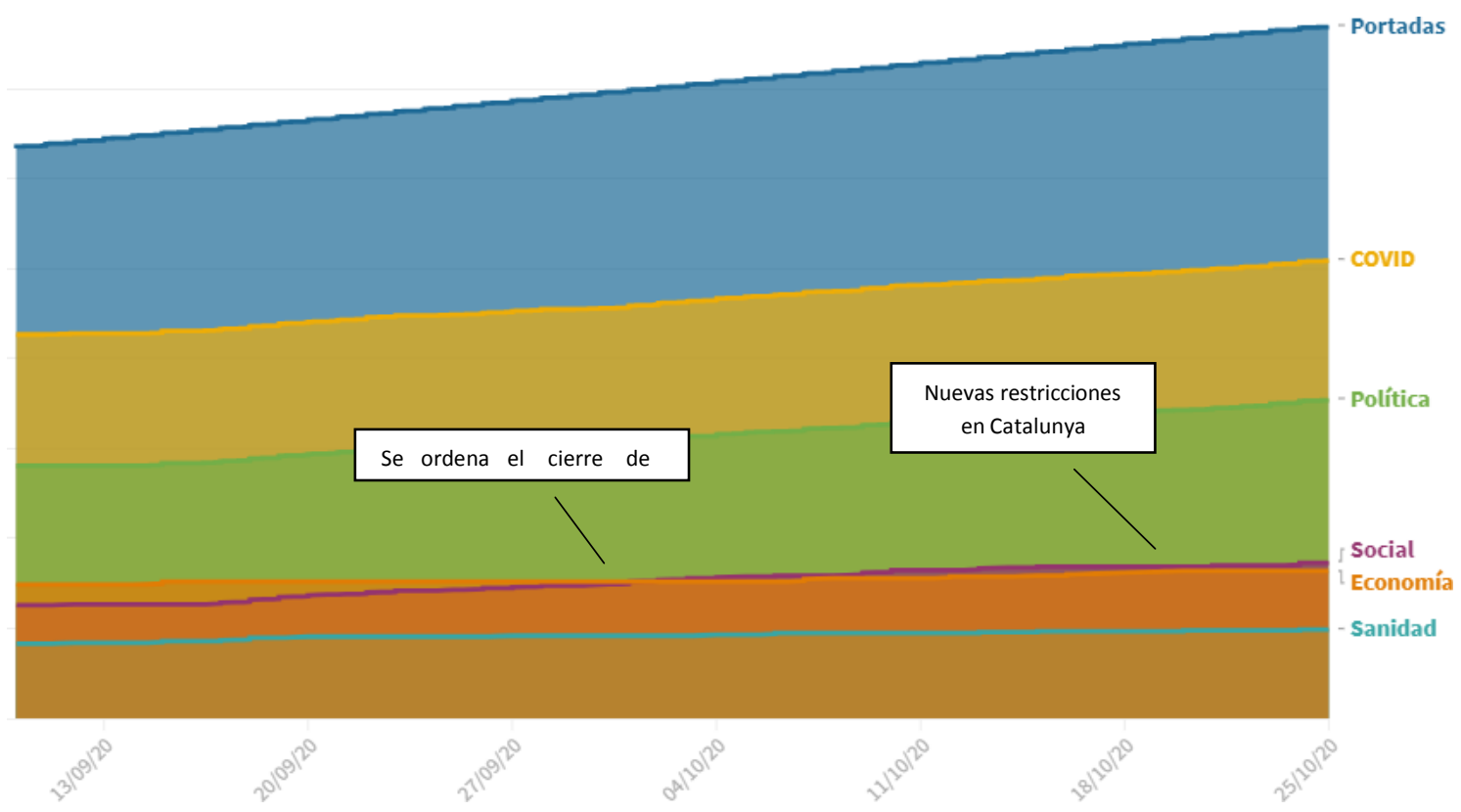

Figura 5. Línea de tiempo con el total de portadas publicadas desde el 10 de septiembre hasta el 25 de octubre y la categorización según tema principal. La agrupación principal fue COVID y dentro de esta: Política, Social, Economía y Sanidad. Fuente: propia

Como se ha comentado anteriormente la temática categorizada como Social superó, a medida que avanzaba la pandemia, a otras subagrupaciones como las referidas a noticias explícitamente sanitarias (Sanidad) y a noticias con contenido económico (Economía). En la figura 5 puede observarse en detalle cómo dos elementos incitaron el aumento de portadas dedicadas a las medidas con afectación social. En primer lugar el confinamiento en Madrid tras diversas polémicas entre el gobierno autonómico y el gobierno central ${ }^{x \times i v}$ y en segundo la ampliación de medidas por parte de la Generalitat de Catalunya debido al aumento de contagios $^{\mathrm{xxv}}$ (que desde una perspectiva geográfica nacional puede observarse en la figura 2). Ahora bien, conviene reseñar que la superación de la temática Social respecto la temática Economía se produce de una forma menos acusada que en el caso de Sanidad. Esto es debido a que de forma simultánea aparecieron noticias referidas a las medidas económicas del Gobierno central ${ }^{\mathrm{x} v \mathrm{i}}$ además de que en esta franja algunas noticias categorizadas como Social cumplían los requisitos para formar parte de la subagrupación Economía ${ }^{x x v i i}$. 
Estas dos alteraciones visualizadas en las figuras 4 y 5 expresan con detalle el aumento de la temática sobre medidas con afectación social en proporción con la presencia en portada de otras temáticas que fueron hegemónicas en la primera ola pero que perdieron protagonismo, al menos en su aspecto informativo, a medida que la pandemia se alargó en el tiempo. Como resultado de esta tendencia la categorización Social se situó como tercer concepto más importante con un 35\% de portadas en las que fue tema principal, por encima de Economía con un $31,5 \%$ y Sanidad con un $20 \%$.

\section{CONCLUSIONES}

Tal como se ha comentado anteriormente esta investigación ha analizado los temas principales de portada de los tres diarios españoles con mayor público y ha tomado como punto de partida el momento en que la pandemia empezó a considerarse por la prensa española como un problema interno. Por esta razón el marco temporal analizado se abrió el 11 de febrero con la portada de La Vanguardia y se cerró con las tres portadas del 7 de noviembre de 2020, fecha en que según Eurostat la segunda ola alcanzó el pico de contagios.

En el apartado de resultados se ha desgranado cómo las portadas de los tres principales diarios españoles pueden considerarse un indicador del grado en que la pandemia por el COVID ha afectado en España. Prueba de ello es la proporción en que este tema ha copado la noticia principal de portada durante la franja analizada, especialmente en los primeros meses en que casi monopolizaron la actualidad informativa.

Las primeras conclusiones que pueden extraerse son, sin embargo, potencialmente sintomáticas de las propias rutinas periodísticas que le son consustanciales a cada medio y sin relación directa con la crisis sanitaria. Por ejemplo que el medio La Vanguardia tenga en su área de interés geográfica Catalunya en comparación con El País y El Mundo pese a que todos ellos son medios de tirada nacional. También la presunta actitud crítica del diario El Mundo, expresada mediante el protagonismo de los partidos de la oposición y el tratamiento de la investigación por el 8-M, sería susceptible de extrapolarse y generalizarse fuera de la pandemia. Aunque esta investigación no puede corroborar semejante aspecto sí que resulta un indicio revelador que reforzaría las conclusiones de trabajos previos durante el inicio de la pandemia especialmente el elaborado por Masip et al. (2020) y de forma más concreta el de 
Sánchez et al. (2020) cuando señalan el uso de los titulares sensacionalistas de El Mundo como forma para criticar al Gobierno.

Si bien, las conclusiones relevantes que se obtienen a través de esta investigación se encuentran vinculadas con dos hallazgos muy significativos. Por una parte la correlación entre cifras de contagios y tratamiento como tema principal de portada. En este aspecto pueden observarse dos fenómenos relacionados con la elasticidad de la curva sobre cobertura mediática: los diarios mantienen el foco en la pandemia durante el verano pese a la mejora del número de contagios (función de alerta) y, aunque los casos se disparen en la segunda ola, no se alcanza la cobertura desplegada en la primera. Resulta llamativa esta segunda señal que puede extraerse de la visualización de datos pues la desviación entre aumento en los contagios de la segunda ola y moderación en el tratamiento informativo podría entenderse como una normalización de la pandemia. La crisis sanitaria perdería peso como acontecimiento principal y se convertiría, en comparación con la primera ola, en una situación añadida a la actualidad informativa. Este resultado confirmaría trabajos previos como el realizado por Pearman, et al. (2021) según el cual la cobertura mundial sobre la pandemia se encuentra en retroceso constante una vez alcanzado el pico en febrero-marzo de 2020.

Este segundo fenómeno que podría definirse como potencial normalización de la pandemia adquiere un valor más notable si se coloca en perspectiva con el otro hallazgo significativo que se ha obtenido en la presente investigación. Como se ha comentado el análisis de contenido generó cuatro temáticas basadas en los titulares de las noticias de portada: Política, Economía, Sanidad y Social. De estas cuatro la subagrupación Social (medidas que afectan a la convivencia), aun estando por debajo de Política, fue la que tuvo una evolución mayor a nivel proporcional llegando a superar a Sanidad (con el inicio de la desescalada) y a Economía (con las primeras restricciones de la segunda ola). Por otro lado la organización jerárquica en los primeros meses (Política, Economía, Sanidad) confirman los resultado del trabajo previo de Gómez et al. (2020).

La reducción del número de temas principales de portada dedicados exclusivamente a la pandemia (primer hallazgo), combinado con la progresión en términos relativos de la temática Social (segundo hallazgo) podría indicar que el tratamiento informativo de los tres diarios 
analizados señalan una mayor preocupación por el estilo de vida durante la pandemia como escenario ineludible durante un tiempo que se ha prolongado. Es decir, según la cobertura en las portadas, la crisis sanitaria podría indicar una pérdida del estatus primigenio como catástrofe sanitaria (temática Sanidad) asumiendo el rol de un limitante a la convivencia (ligeramente por encima de un condicionante económico). Por tanto, es posible concluir que el tratamiento informativo en diarios de referencia se ha desplazado según ha avanzado la pandemia en España. Desde una preeminencia casi absoluta (primera ola), a una advertencia mantenida (fase sin contagios) hasta una normalización parcial con importante peso de las afectaciones a la vida en sociedad (segunda ola). En todo caso, serán bienvenidas investigaciones futuras cuya perspectiva permita una visión global del tratamiento informativo sobre el mundo en el que nos ha tocado vivir.

\section{REFERENCIAS}

ABUÍN-VENCES, N., SIERRA-SÁNCHEZ, J., MAÑAS-VINIEGRA, L. y NÚÑEZ-GÓMEZ, P. (2020). Tratamiento informativo de la pandemia del coronavirus en los medios digitales españoles. Hipertext. net, (21), 15-26. https://doi.org/10.31009/hipertext.net.2020.i21.02

ALEIXANDRE-BENAVENT, R., CASTELLÓ-COGOLLOS, L. y VALDERRAMA-ZURIÁN, J. C. (2020). Información y comunicación durante los primeros meses de Covid-19. Infodemia, desinformación y papel de los profesionales de la información. El profesional de la información (EPI), 29(4). https://doi.org/10.3145/epi.2020.jul.08

ALVARADO, H. (2008). El rostro de la prensa de calidad. La información internacional desde la portada de los grandes diarios europeos. Textual \& Visual Media, 1, 21-47. Recuperado de https://bit.ly/3nZstpH el 24 de diciembre de 2020

ANSCOMBE, F. J. (1973). Graphs in statistical analysis. The american statistician, 27(1), 17-21. https://doi.org/10.2307/2682899

ARCAVI, A. (2003). The role of visual representations in the learning of mathematics. Educational studies in mathematics, 52(3), 215-241. 
https://doi.org/10.1023/A:1024312321077

CASERO-RIPOLLÉS, A. (2020). Impacto del Covid-19 en el sistema de medios. Consecuencias comunicativas y democráticas del consumo de noticias durante el brote. El profesional de la información (EPI), 29(2). http://orcid.org/0000-0001-69864163

CASTILlO-ESPARCIA, A., FERNÁNDEZ-SOUTO, A. B. y PUENTES-RIVERA, I. (2020). Comunicación política y Covid-19. Estrategias del Gobierno de España//Political communication and Covid-19: strategies of the Government of Spain. Profesional de la información, 29(4). https://doi.org/10.3145/epi.2020.jul.19

DANIELSON, W. A. y LASORSA, D. L. (2020). Perceptions of social change: 100 years of frontpage content in The New York Times and The Los Angeles Times. En Text analysis for the social sciences: Methods for drawing statistical inferences from texts and transcripts, 103. https://doi.org/10.4324/9781003064060-7_

EDELMAN (2020). "Brand trust and the coronavirus pandemic". Recuperado de https://bit.ly/38xVv99 el 24 de diciembre de 2020

GODINO, J. D., GONZATO, M., CAJARAVILLE, J. A. y FERNÁNDEZ, T. (2012). Una aproximación ontosemiótica a la visualización en educación matemática. Enseñanza de las ciencias: revista de investigación y experiencias didácticas, 109-130. Recuperado de https://bit.ly/2JjZ4Y4 el 24 de diciembre de 2020

GÓMEZ, P. N., VENCES, N. A., SÁNCHEZ, J. S. y VINIEGRA, L. M. (2020). El enfoque de la prensa española durante la crisis del Covid-19. Un análisis del framing a través de las portadas de los principales diarios de tirada nacional. Revista Latina de Comunicación Social, (78), 41-63. https://doi.org/10.4185/rlcs-2020-1468_

KRIPPENDORFF, K. (2018). Content analysis: An introduction to its methodology. Thousand Oaks: Sage

LÁZARO-RODRÍGUEZ, P. y HERRERA-VIEDMA, E. (2020). Noticias sobre Covid-19 y 2019- 
nCoV en medios de comunicación de España: el papel de los medios digitales en tiempos de confinamiento. El profesional de la información (EPI), 29(3). https://doi.org/10.3145/epi.2020.may.02

MACNAMARA, J. (2018). 11 Content Analysis. En P. M. Napoli (Ed.), Mediated Communication (pp. 191-212). Berlin, Boston: De Gruyter

MASIP, P., ARAN-RAMSPOTT, S., RUIZ-CABALLERO, C., SUAU, J., ALMENAR, E. y PUERTAS-GRAELL, D. (2020). Consumo informativo y cobertura mediática durante el confinamiento por el Covid-19: sobreinformación, sesgo ideológico y sensacionalismo. El profesional de la información (EPI), 29(3). https://doi.org/10.3145/epi.2020.may.12

MOTTA, M., STECULA, D. y FARHART, C. (2020). How right-leaning media coverage of COVID-19 facilitated the spread of misinformation in the early stages of the pandemic in the US. Canadian Journal of Political Science/Revue canadienne de science politique, 1-8. https://doi.org/10.31235/osf.io/a8r3p

NEUENDORF, K. A. (2017). The content analysis guidebook. Thousand Oaks: Sage.

NIELSEN, R. K., FLeTCHER, R., NEWMAN, N., BRENNEN, S. J. y HOWARD, P. N. (2020). Navigating the 'infodemic': How people in six countries access and rate news and information about coronavirus. Reuters Institute. Recuperado de https://bit.ly/3plHZfw el 24 de diciembre de 2020

PEARMAN, O., BOYKOFF, M., OSBORNE-GOWEY, J., AOYAGI, M., BALLANTYNE, A. G., CHANDLER, P.,... YTTERSTAD, A. (2021). COVID-19 media coverage decreasing despite deepening crisis. The Lancet Planetary Health, 5(1), e6-e7. https://doi.org/10.1016/S2542-5196(20)30303-X

ROTHKOPF, D. J. (2003). 'When the Buzz Bites Back', Washington Post, 11 de mayo, p. B01

SÁNCHEZ, L. P., DE FRUTOS TORRES, B. y MARTíN, A. G. (2020). La COVID-19 en la prensa española. Encuadres de alarma y tranquilidad en las portadas de El País, El 
Mundo y La Vanguardia. Revista de Comunicación y Salud, 10(2), 355-384.

XIFRA, J. (2020). Comunicación corporativa, relaciones públicas y gestión del riesgo reputacional en tiempos del Covid-19. El profesional de la información (EPI), 29(2). https://doi.org/10.3145/epi.2020.mar.20

i Pese a su uso extendido el término infodemia no está reconocido completamente en la investigación en ciencias sociales (Nielsen, Fletcher, Newman, Brennen y Howard, 2020). Puede definirse como epidemias de información difundidas por las nuevas tecnologías que combinan algo de realidad con rumores, suposiciones y preocupación (Rothkopf, 2003). ii Esta investigación indicó que respecto la información sobre la crisis sanitaria los medios nacionales ofrecen la mayor credibilidad para los encuestados. Por ejemplo sólo una porción muy pequeña de la audiencia puso en duda una información aparecida únicamente en un medio nacional en comparación con la escasa confianza que generaron los contenidos sobre el COVID publicados específicamente por influencers o en redes sociales en general.

iii Lectores: 893.000 (dato EGM). Actualizado el 16 de diciembre de 2020

iv Lectores: 621.000 (dato EGM). Actualizado el 16 de diciembre de 2020

$v$ Lectores: 454.000 (dato EGM). Actualizado el 16 de diciembre de 2020

${ }^{v i}$ Pese a que hubo portadas anteriores referidas a los efectos del COVID el primer tema principal de portada que relacionaba la pandemia con su potencial afectación en territorio español fue de La Vanguardia: "El Mobile se mantiene firme pese a la baja de empresas".

vii Según datos del Centro Europeo para la Prevención y Control de Enfermedades. Consultado el 16 de diciembre en https://elpais.com/sociedad/2020-12-03/espana-es-uno-de-los-paiseseuropeos-con-menos-contagios-en-una-segunda-ola-a-distintas-velocidades.html viii Estos autores analizaron las portadas de los seis periódicos más importantes de tirada nacional durante los primeros meses de pandemia y observaron mediante la teoría del framing elementos significativos como el tono de la información.

${ }^{i x}$ Estos autores analizaron las portadas de los mismos diarios que el presente trabajo pero tomando los primeros meses de la pandemia y se centraron en si se transmitían mensajes de alarma o de tranquilidad en relación a un enfoque sensacionalista o con interés político. 
× Un mismo titular podría estar categorizado en varias temáticas si cumplía las características expresadas en los criterios.

${ }^{x i}$ A razón de 3 portadas por día menos el primer día del marco de investigación en el que no se computaron las dos portadas no referidas al COVID de esta forma se tomó como punto de partida una única portada esa fecha.

xii La sede de La Vanguardia se encuentra en Barcelona, capital de Catalunya. Recuperado de https://www.lavanguardia.com/contacto.html

xiii El País publicó la investigación como tema de portada sin mencionar al Ministro del Interior y su enfoque resaltó que la acción del gobierno no estaba siendo considerada delictiva por parte de la justicia. La Vanguardia por su parte informó sobre el cierre de la investigación.

${ }^{\text {xiv } L a ~ i n v e s t i g a c i o ́ n ~ f u e ~ a r c h i v a d a ~ e n ~ p r i m e r a ~ i n s t a n c i a ~ e l ~} 12$ de junio del 2020 y la cobertura informativa se inició a finales de mayo. Recuperado el 16 de diciembre de 2020 de https://www.eldiario.es/politica/archiva-investigacion-delegado-gobierno-

madrid_1_6020976.html

${ }^{x v}$ La investigación de Gómez et al. (2020) señaló, sin embargo, que durante los primeros meses de pandemia El Mundo fue el medio con mayor número de portadas positivas sobre la pandemia.

${ }^{\text {xvi }}$ Recuperado el 16 de diciembre de 2020 de https://data.europa.eu/euodp/en/data/dataset/covid-19-coronavirus-data xvii "El juez del 'caso Dina' pide al Supremo que investigue a Iglesias" (El País, 8 de octubre) xviii "Los expertos denuncian el socavamiento de la corona desde Moncloa" (El Mundo, 12 de octubre)

xix "La reforma por la vía rápida del Poder Judicial complica los fondos europeos" (El País 14 de octubre)

xx "El Supremo confirma que el PP se lucró con la trama Gürtel" (El País, 15 de octubre)

xxi "Trump pide el recuento de votos ante el avance de Biden" (La Vanguardia, 5 de noviembre) xxii También durante el mes de octubre se produjo la moción de censura al Gobierno por parte de Vox (“EI PP decidirá a última hora su voto en la moción de censura de Vox", La Vanguardia, 19 de octubre). No obstante el texto de la moción argumentaba, entre otros asuntos, que la 
gestión de la pandemia por parte del Gobierno fue ineficiente por lo que este tema se categorizó como COVID.

xxiii La metodología aplicada por estos autores no incluyó la temática Social y los conceptos asociados se subsumieron dentro del resto.

xxiv "Madrid se rebela contra el plan de Sanidad de confinar la capital" (El País, 1 de octubre); "Cerrojazo a Madrid con miedo al "caos" y a la crisis" (El Mundo, 3 de octubre)

xxv "Catalunya aconseja volver al teletrabajo quince días" (La Vanguardia, 12 de octubre)

xxvi "Récord de deuda, déficit y gasto sin garantías de recuperación" (El Mundo, 7 de octubre)

xxvii "El Govern sopesa el cierre de bares y restaurantes 15 días" (La Vanguardia, 14 de noviembre) 